\title{
Dialysis in Northern India during COVD-19 pandemic: trying to maintain a balance
}

\author{
Abhilash Chandra ${ }^{1}$ (D) Namrata Rao ${ }^{1} \cdot$ Divya Srivastava $^{2}$
}

Received: 4 May 2020 / Accepted: 25 May 2020 / Published online: 1 June 2020

(c) Springer Nature B.V. 2020

Editor,

Spread of severe acute respiratory syndrome coronavirus 2 (SARS-CoV-2) in India in March 2020 marked the onset of a new unseen era of lockdown starting from 25th March. It brought with itself a new set of rules and regulations [1]. Emergency Medical services were exempted from it. A heightened response system was initiated to counter this new unprecedented situation.

One of the most significant challenge was the continuation of dialysis programme. Cessation of public transport made movement of private vehicles a norm to commute to dialysis centres. Those unable to afford one were offered ambulances by the government agencies, albeit it wasn't an easy affair because of skewed demand and supply ratio. Financial insufficiencies precipitated by halted economic activities, added to the woes.

Free movement of all the vehicles carrying medical supplies was allowed on priority basis [1]. This enabled dialysis centres to maintain an adequate inventory of the consumables. All the dialysis staff were trained for donning, doffing of personal protective equipment (PPE) and handling of COVID-19 positive and suspected patients. Achilles heel, however, was the availability of PPE which was scarce. At times surgical gowns, masks and gloves were the only available ones.

Abhilash Chandra

acn393@gmail.com

Namrata Rao

jrambo3636@gmail.com

Divya Srivastava

dr.divshri@gmail.com

1 Department of Nephrology, Dr. RMLIMS, Vibhuti Khand, Gomti Nagar, Lucknow 226010, India

2 Department of Anaesthesiology, SGPGIMS, Rae Bareli Road, Lucknow 226014, India
Screening of patients as well as their attendants for COVID-19 symptoms, travel and contact history before entering the dialysis unit was routinely done [2]. In view of the high percentage of asymptomatic patients (>50\%) [3] affected with Cov-2, everybody entering the unit was asked to wear mask (preferably three layered) and clean hands with soap and water/alcohol-based sanitizer. Posters carrying information about the disease were displayed outside the dialysis unit. Regular cleaning of the unit with $1 \%$ hypochlorite solution after every shift was done. Surprise checks were conducted to look for any lacunae and they were corrected.

Despite these measures outbreak of COVID-19 was reported in a few centres [4]. The centres were sanitized and contact staff was quarantined. To prevent spread, all the dialysis patients were screened for Cov-2 (symptoms and test if required), before shifting them to other units. The affected units were unable to carry on with schedules due to scarcity of trained technicians after quarantine of some of them for 14 days.

Separate dialysis units were established in hospitals dedicated for COVID patients. Infrastructure comprised of hemodialysis and portable reverse osmosis machines. Staff on duty were placed on a schedule of one-week work followed by a two-week quarantine at these centres [5].

Patients on peritoneal dialysis (PD) suffered a different plight. Not requiring frequent visits to hospital in times when travelling was financially taxing, was a boon. But availability of PD fluids at selected medical shops/hospitals meant difficulty in procuring these. The prohibition on movement imposed by the government made the task further difficult. The dedicated PD nurses were assigned the job of contacting all PD patients to ensure accessibility to PD fluid and guide them to the nearest available distributor. Together with the consultants they also helped the patients overcome any associated PD complication. New PD initiations were almost on hold except for those with complete vascular access failure.

Consultations were provided primarily on telephone. Hospital visits were limited to emergencies. Tele medicine 
was initiated in a couple of centres. But limited access to internet in the rural population was a hindrance in this regard [6].

Looking at the silver lining in the cloud, the current scenario provided an opportunity to work upon the possibility of distant consultation and thereby reducing the overburdened OPDs and public conveyance system even after the crisis wanes out.

\section{References}

1. https://www.mohfw.gov.in/pdf/Annexure_MHA.pdf. Accessed 24 Mar 2020
2. https://www.mohfw.gov.in/pdf/RevisedGuidelinesforDialysisof COVID19Patients.pdf. Accessed 7 Apr 2020

3. Nishiura $H$ (2020) Estimation of the asymptomatic ratio of novel coronavirus infections (COVID-19). Int J Infect Dis 94:154-155

4. https://www.hindustantimes.com/delhi-news/how-covid-19-isaffecting-dialysis-patients/story-zKXDs Vd50rrKoY4HrXKL VN.html. Accessed 21 Apr 2020

5. https://www.rahatup.in/Home/UPGovernmentOrder?Upgov _Id=2\&DepartmentId=5. Accessed 3 Apr 2020

6. https://trai.gov.in/sites/default/files/PIR_08012020_0.pdf. Accessed 8 Jan 2020

Publisher's Note Springer Nature remains neutral with regard to jurisdictional claims in published maps and institutional affiliations. 